\title{
Quantum-Limited Directional Amplifiers with Optomechanics
}

\author{
Daniel Malz, ${ }^{1}$ László D. Tóth, ${ }^{2}$ Nathan R. Bernier, ${ }^{2}$ \\ Alexey K. Feofanov, ${ }^{2}$ Tobias J. Kippenberg, ${ }^{2}$ and Andreas Nunnenkamp ${ }^{1}$ \\ ${ }^{1}$ Cavendish Laboratory, University of Cambridge, Cambridge CB3 OHE, United Kingdom \\ ${ }^{2}$ Institute of Physics, École Polytechnique Fédérale de Lausanne, Lausanne 1015, Switzerland
}

(Received 28 April 2017; revised manuscript received 15 August 2017; published 8 January 2018)

\begin{abstract}
Directional amplifiers are an important resource in quantum-information processing, as they protect sensitive quantum systems from excess noise. Here, we propose an implementation of phase-preserving and phase-sensitive directional amplifiers for microwave signals in an electromechanical setup comprising two microwave cavities and two mechanical resonators. We show that both can reach their respective quantum limits on added noise. In the reverse direction, they emit thermal noise stemming from the mechanical resonators; we discuss how this noise can be suppressed, a crucial aspect for technological applications. The isolation bandwidth in both is of the order of the mechanical linewidth divided by the amplitude gain. We derive the bandwidth and gain-bandwidth product for both and find that the phasesensitive amplifier has an unlimited gain-bandwidth product. Our study represents an important step toward flexible, on-chip integrated nonreciprocal amplifiers of microwave signals.
\end{abstract}

DOI: 10.1103/PhysRevLett.120.023601

Introduction.-Nonreciprocal transmission and amplification of signals are essential in communication and signal processing, as they protect the signal source from extraneous noise. Conventional ferrite-based devices rely on magnetic fields and are challenging to integrate in superconducting circuits. Hence, there exists strong incentive to find more suitable implementations [1-14]. In the microwave domain, the strong Josephson nonlinearity and parametric pumping can achieve both photon gain and conversion processes, which have been exploited to realize circulators and directional amplifiers [5,13-15]. Another promising platform is optomechanics, where nonreciprocal devices [16-27], phase-preserving amplifiers [28-32], and phase-sensitive amplifiers [33-36] have been proposed and realized.

In recent theoretical work, Ranzani and Aumentado $[16,17]$ analyzed general conditions for nonreciprocity in parametrically coupled systems, and showed that nonreciprocity arises due to dissipation in ancillary modes and multipath interference. Metelmann and Clerk [18] have shown that any coherent interaction can be made directional by balancing it with a dissipative process. Indeed, this insight led to a demonstration of nonreciprocity using optomechanics in the optical domain [19], and theoretical investigations into minimal implementations of directional amplifiers [20].

While implementing the balance between a direct coherent coupling between the cavities and a dissipative interaction is challenging experimentally, Refs. [25-27] have recently studied and demonstrated nonreciprocal transmission between two cavity modes where two mechanical resonators each mediate both coherent and dissipative coupling. Here, building on this concept, we propose directional amplifiers using exclusively optomechanical interactions. Microwave tones on the red and blue sidebands enable so-called beam-splitter and two-mode squeezing interactions (cf. Fig. 1), leading to a total of eight controllable terms in the Hamiltonian. We identify and analyze a simple directional phase-preserving amplifier that uses four tones and a directional phase-sensitive amplifier using six tones. While the gain-bandwidth product of

(a)

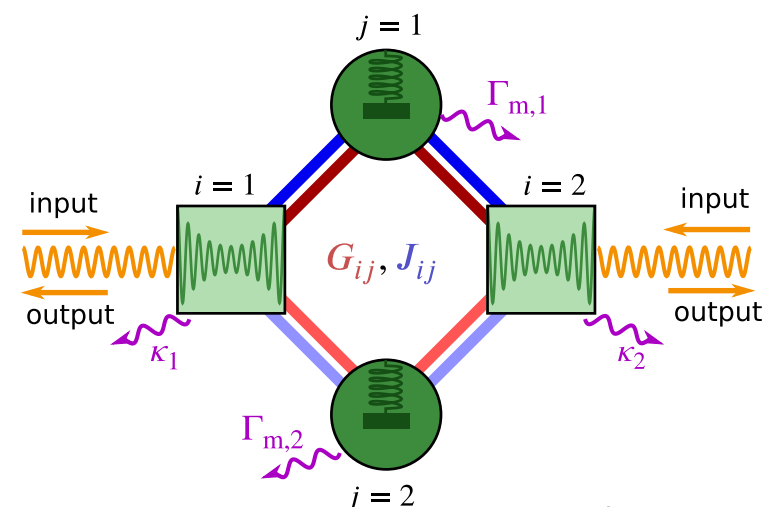

(b)

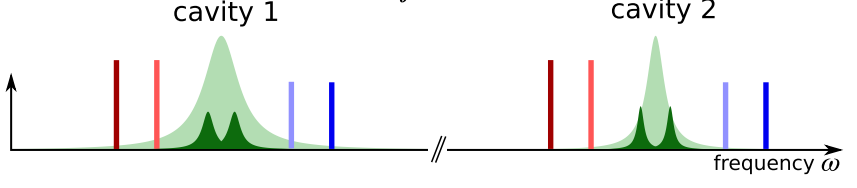

FIG. 1. (a) Schematic of all possible interactions in optomechanical plaquette comprising two mechanical resonators (round, dark green) and two cavities (square, light green). The cavities [light-green Lorentzians in (b)] are driven by up to eight tones, placed close to the mechanical sidebands, at frequencies $\omega_{c, i} \pm\left(\Omega_{j}+\delta_{j}\right)$, as illustrated in (b), which induce hopping and two-mode squeezing interactions $\left(G_{i j}, J_{i j}\right)$, denoted in (a) by red and blue lines connecting the modes. This leads to the timeindependent Hamiltonian (1). 


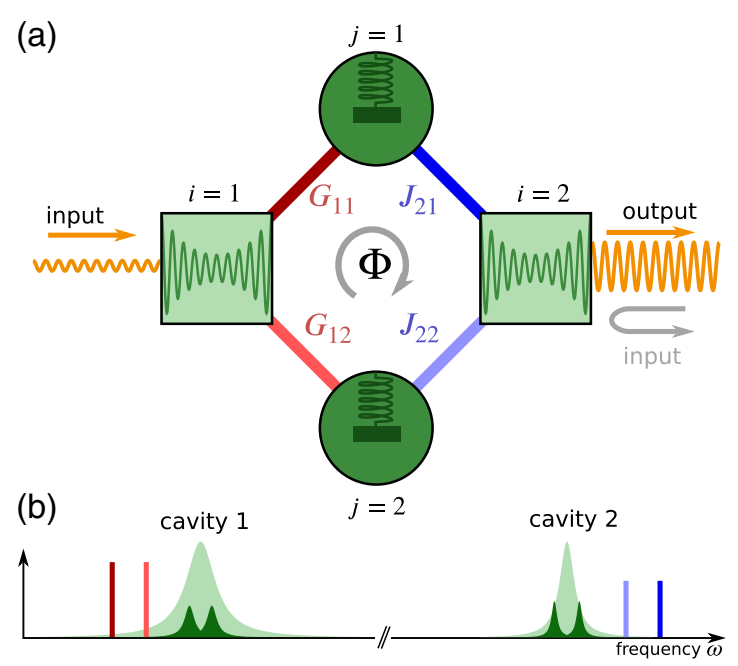

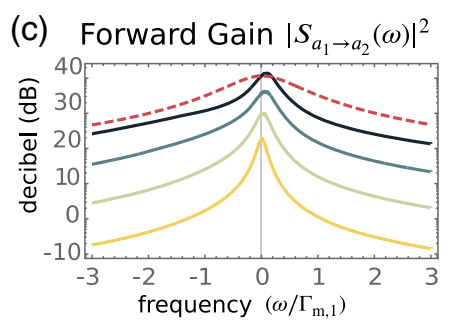

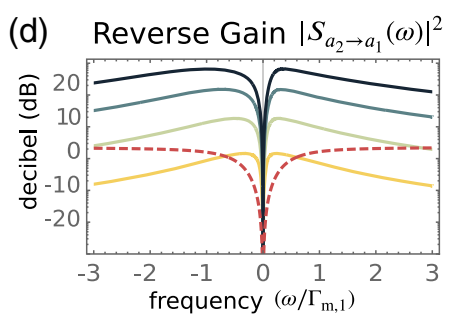

(e)

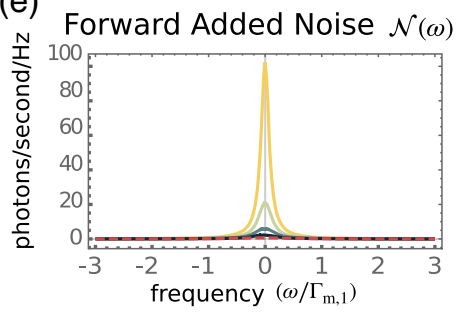

(f) Backward Noise Spectrum

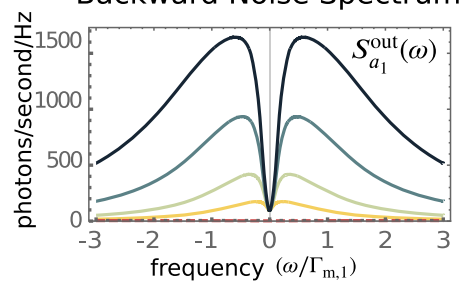

FIG. 2. The directional phase-preserving amplifier. (a) Model: red hopping interactions are impedance matched, blue provide amplification. (b) Pump setup: cavity 1 (cavity 2) is pumped on the red (blue) sidebands of the mechanical resonators. (c)-(f) Plots of forward gain, reverse gain [Eq. (7)], added noise [Eq. (9)], and the output noise fluctuation spectrum of cavity 1, all as functions of frequency in units of $\Gamma_{m, 1}$, for cooperativities $\mathcal{C}_{1}=\{1,3,10,30\}, \mathcal{C}_{2}=\mathcal{C}_{1}-0.1 \sqrt{\mathcal{C}_{1}}$ (yellow to black, or light to dark). Parameters are $\kappa_{2} / \kappa_{1}=0.7, \Gamma_{m, 1} / \kappa_{1}=10^{-2}, \Gamma_{m, 2} / \Gamma_{m, 1}=0.8$, thermal occupation of the mechanical resonators $n_{m, 1}=n_{m, 2}=100$, and cavities $n_{c, 1}=n_{c, 2}=0$. Depending on parameters, external sideband cooling with an auxiliary mode can achieve $n_{m, j} \approx 0$, without negatively affecting amplification properties, as discussed below and in the SM [39]. The red (dashed) curve in each plot illustrates this case, with $\mathcal{C}_{1}=30$ and effective parameters $n_{\mathrm{eff}, i}=n_{m, i}\left(\Gamma_{m, i} / \Gamma_{\mathrm{eff}, i}\right), \Gamma_{\mathrm{eff}, i}=50 \Gamma_{m, i}$.

the phase-preserving amplifier is limited to the cavity decay rate, the phase-sensitive amplifier has an unlimited gainbandwidth product. Both amplifiers can reach their quantum limits of a half and zero added quanta, respectively, and emit thermal noise from the mechanical resonators in the reverse direction, a necessary consequence of impedance matching and directionality. This noise can be reduced through additional sideband cooling without interfering with directionality or amplification. Our proposal bridges the gap between previous theoretical studies and experimental realization.

Model.-We consider an optomechanical plaquette, comprising two microwave cavities coupled via two mechanical resonators [Fig. 1(a)]. The cavities are driven close to the motional sidebands. With a standard treatment $[37,38]$, we derive the time-independent Hamiltonian $(\hbar=1)$

$H_{\mathrm{sys}}=-\sum_{i=1}^{2} \delta_{i} b_{i}^{\dagger} b_{i}-\sum_{i, j=1}^{2} a_{i}^{\dagger}\left(G_{i j} b_{j}+J_{i j} b_{j}^{\dagger}\right)+$ H.c.

where $a_{i}\left(b_{i}\right)$ is the annihilation operator for the $i$ th cavity mode (mechanical resonator), $G_{i j}=\alpha_{i j-} g_{0, i j}$ and $J_{i j}=$ $\alpha_{i j+} g_{0, i j}$ are field-enhanced optomechanical coupling strengths, $\alpha_{i j \pm}$ is the amplitude of the coherent state produced in cavity $i$ due to a pump at frequency $\omega_{c, i} \pm\left(\Omega_{j}+\delta_{j}\right)$, and $g_{0, i j}$ are the vacuum optomechanical couplings. Since the couplings $G_{i j}, J_{i j}$ depend on the pumps, their amplitude and phase are controllable. The interactions are represented in Figs. 1(a), 2(a), and 3(a) as red $\left(G_{i j}\right)$ and blue $\left(J_{i j}\right)$ lines. Further details can be found in the Supplemental Material
(SM) [39], including a discussion about the limits of validity of the rotating-wave approximation (RWA).

We describe the system with quantum Langevin equations [38,40,41]. Neglecting mechanical noise (analyzed later), and eliminating the mechanical modes, we obtain

$$
\sum_{j=1}^{2}\left[\chi_{\mathrm{c}, i}^{-1}(\omega) \delta_{i j}+i \mathbf{T}_{i j}(\omega)\right] \vec{A}_{j}(\omega)=\sqrt{\kappa_{i}} \vec{A}_{i, \text { in }}(\omega)
$$

where the susceptibility $\chi_{\mathrm{c}, i}(\omega)=\left[\kappa_{i} / 2-i \omega\right]^{-1}, \vec{A}_{j(\text {,in })}=$ $\left(a_{j(\text {,in })}, a_{j(\text {,in })}^{\dagger}\right)^{T}$, and each $i \mathbf{T}_{i j}$ is a $2 \times 2$ matrix

$$
\begin{aligned}
i \mathbf{T}_{i j}(\omega)= & \sum_{k=1}^{2} \sigma_{z}\left[\chi_{m, k}(\omega)\left(\begin{array}{cc}
G_{i k} G_{j k}^{*} & G_{i k} J_{j k} \\
J_{i k}^{*} G_{j k}^{*} & J_{i k}^{*} J_{j k}
\end{array}\right)\right. \\
& \left.-\chi_{m, k}^{*}(-\omega)\left(\begin{array}{cc}
J_{i k} J_{j k}^{*} & J_{i k} G_{j k} \\
G_{i k}^{*} J_{j k}^{*} & G_{i k}^{*} G_{j k}
\end{array}\right)\right]
\end{aligned}
$$

where $\sigma_{z}=\operatorname{diag}(1,-1)$ and $\chi_{m, i}(\omega)=\left[\Gamma_{m, i} / 2-i\left(\omega+\delta_{i}\right)\right]^{-1}$. $i \mathbf{T}_{i i}$ is akin to a self-energy for mode $A_{i}$, whereas $i T_{i j}$ for $i \neq j$ is a matrix of coupling strengths between the modes. Since the interaction is mediated by mechanical resonators, their susceptibility $\chi_{m, i}$ appears in the coupling matrix.

Using the input-output relation $a_{i, \mathrm{out}}=a_{i \text {,in }}-\sqrt{\kappa_{i}} a_{i}$ [40], the optical scattering matrix is $\mathbf{S}_{\text {optical }}(\omega)=\mathbb{1}_{4}-\mathbf{L} \chi(\omega) \mathbf{L}$, where $\mathbf{L}=\operatorname{diag}\left(\sqrt{\kappa_{1}}, \sqrt{\kappa_{1}}, \sqrt{\kappa_{2}}, \sqrt{\kappa_{2}}\right)$, and 


$$
[\chi(\omega)]^{-1}=\left(\begin{array}{cc}
\chi_{\mathrm{c}, 1}^{-1}(\omega) \mathbb{1}_{2}+i \mathbf{T}_{11}(\omega) & i \mathbf{T}_{12}(\omega) \\
i \mathbf{T}_{21}(\omega) & \chi_{\mathrm{c}, 1}^{-1}(\omega) \mathbb{1}_{2}+i \mathbf{T}_{22}(\omega)
\end{array}\right) .
$$

The system is nonreciprocal if the moduli of forward- and reverse-scattering amplitudes differ, which occurs if $\left|\mathbf{T}_{12}\right| \neq\left|\mathbf{T}_{21}\right|$. Looking for instance at the top left elements $\left[i T_{12}\right]_{11},\left[i T_{21}\right]_{11}$, we see that nonreciprocity arises because flipping direction $(1 \leftrightarrow 2)$ conjugates the complex couplings, but leaves the mechanical susceptibility unchanged. Nonreciprocity can also be understood in the framework presented in Ref. [18] [39].

Directional phase-preserving amplifier (DPPA).-We consider the coupling amplitudes [cf. Fig. 2(a)]

$$
\begin{aligned}
\mathbf{G} & =\frac{1}{2}\left(\begin{array}{cc}
e^{i \Phi / 2} \sqrt{\mathcal{C}_{1} \Gamma_{m, 1} \kappa_{1}} & e^{-i \Phi / 2} \sqrt{\mathcal{C}_{1} \Gamma_{m, 2} \kappa_{1}} \\
0 & 0
\end{array}\right), \\
\mathbf{J} & =\frac{1}{2}\left(\begin{array}{cc}
0 & 0 \\
\sqrt{\mathcal{C}_{2} \Gamma_{m, 1} \kappa_{2}} & \sqrt{\mathcal{C}_{2} \Gamma_{m, 2} \kappa_{2}}
\end{array}\right),
\end{aligned}
$$

that is, the first (second) cavity has two drives, close to the red (blue) motional sidebands corresponding to the mechanical resonators [cf. Figs. 2(a) and 2(b)]. We have already written the amplitudes in terms of cooperativities $\mathcal{C}_{1 i}=4\left|G_{1 i}\right|^{2} /\left(\kappa_{1} \Gamma_{m, i}\right), \mathcal{C}_{2 i}=4\left|J_{2 i}\right|^{2} /\left(\kappa_{2} \Gamma_{m, i}\right)$, and chosen them to be equal in both arms, $\mathcal{C}_{1} \equiv \mathcal{C}_{1 i}, \mathcal{C}_{2} \equiv \mathcal{C}_{2 i}$. Given Eqs. (2) and (5), isolation $\left(\mathbf{T}_{12}=\mathbf{0}\right)$ requires $\delta_{1}^{2} \Gamma_{m, 2}^{2}=$ $\delta_{2}^{2} \Gamma_{m, 1}^{2}$ [42]. $\delta_{1}$ and $\delta_{2}$ must have opposite signs, and we parametrize them by a single dimensionless variable $\delta_{1}=\delta \Gamma_{m, 1}, \delta_{2}=-\delta \Gamma_{m, 2}$.

Isolation occurs for certain phases of the coupling amplitudes $\theta_{1 i} \equiv \arg \left(G_{1 i}\right)$ and $\theta_{2 i} \equiv \arg \left(J_{2 i}\right)$. However, only the overall relative "plaquette phase," $\Phi \equiv \theta_{11}+$ $\theta_{21}-\theta_{12}-\theta_{22}$, is relevant, which explains the parameterization in Eq. (5). Setting $\delta=\sqrt{2 \mathcal{C}_{1}-1} / 2$ achieves impedance matching (i.e., vanishing reflection at cavity 1 ), attainable for $\mathcal{C}_{1} \geq 0.5$. Then the plaquette phase at which isolation occurs is

$$
\Phi=i \log \left(\frac{2 \delta-i}{2 \delta+i}\right)=2 \arccos \sqrt{1-1 /\left(2 \mathcal{C}_{1}\right)} .
$$

Inverting the plaquette phase $-\Phi$ leads to isolation in the opposite direction [39].

We have chosen the couplings [Eq. (5)] due to the following reasons. First, an even number of blue and red tones ensures equivalent arms of the circuit. Second, amplification requires blue tones. Third, a directional amplifier with four blue tones cannot be impedance matched to the signal source [39]. Last, swapping hopping and amplifier interactions in one arm of the circuit cannot lead to directional amplification [43].

The condition $\mathcal{C}_{2}<\mathcal{C}_{1}$ ensures that the system does not exceed the parametric instability threshold. In the limit of large gain, we obtain our first main result, the scattering matrix (on resonance)

$$
\left(\begin{array}{c}
a_{1, \text { out }} \\
a_{2, \text { out }}^{\dagger}
\end{array}\right)=\left(\begin{array}{cccc}
\frac{1}{\sqrt{2}} & -\frac{1}{\sqrt{2}} & 0 & 0 \\
\frac{i \sqrt{\mathcal{G}}}{\sqrt{4 \mathcal{C}_{1}}} & \frac{i \sqrt{\mathcal{G}}}{\sqrt{4 \mathcal{C}_{1}}} & -\sqrt{\mathcal{G}} & \frac{\mathcal{C}_{1}+\mathcal{C}_{2}}{\mathcal{C}_{2}-\mathcal{C}_{1}}
\end{array}\right)\left(\begin{array}{l}
b_{1, \text { in }} \\
b_{2, \text { in }} \\
a_{1, \text { in }} \\
a_{2, \text { in }}^{\dagger}
\end{array}\right),
$$

with vanishing reverse gain $\left|S_{a_{2} \rightarrow a_{1}}(0)\right|^{2}$, but forward gain

$$
\left|S_{a_{1} \rightarrow a_{2}}(0)\right|^{2} \equiv \mathcal{G}=\frac{4 \mathcal{C}_{1} \mathcal{C}_{2}}{\left(\mathcal{C}_{1}-\mathcal{C}_{2}\right)^{2}},
$$

which can in principle be arbitrarily large, as long as the RWA is valid [39].

At the same time, thermal noise from the mechanical resonators is suppressed by increasing $\mathcal{C}_{1}$, as is demonstrated in Fig. 2(e), where we plot the noise added to the signal $\mathcal{N}(\omega)=\mathcal{G}^{-1} \sum_{i \neq a_{1}}\left(n_{i}+1 / 2\right)\left|S_{i \rightarrow a_{2}}(\omega)\right|^{2}[30,37,44]$, where we sum over all noise sources, with associated thermal occupation $n_{i}$ and scattering amplitude to the second cavity $S_{i \rightarrow a_{2}}$. Using Eq. (7), and denoting thermal cavity (mechanical) occupations by $n_{c, i}\left(n_{m, i}\right)$, the noise on resonance

$\mathcal{N}_{\mathrm{DPPA}}=\frac{1}{4 \mathcal{C}_{1}}\left(n_{m, 1}+n_{m, 2}+1\right)+\frac{\left(\mathcal{C}_{1}+\mathcal{C}_{2}\right)^{2}}{4 \mathcal{C}_{1} \mathcal{C}_{2}}\left(n_{c, 2}+\frac{1}{2}\right)$.

As a result, for large $\mathcal{C}_{1} \gtrsim \mathcal{C}_{2}$, and vanishing thermal occupation of the cavity input, we reach the quantum limit of half a quantum of added noise, $\mathcal{N}_{\text {DPPA }} \rightarrow 1 / 2$ [37,44].

Another important figure of merit is noise emerging from cavity 1 , characterized by the output noise spectral density, $S_{a_{1}}^{\text {out }}(\omega) \equiv \int\left(d \omega^{\prime} / 2 \pi\right)\left\langle a_{1, \text { out }}^{\dagger}(\omega) a_{1, \text { out }}\left(\omega^{\prime}\right)\right\rangle$, which we plot in Fig. 2(f). Ultimately, the reason for building directional amplifiers is to reduce this figure. On resonance, $S_{1, \text { out }}^{\text {DPPA }}(0)=\left(n_{m, 1}+n_{m, 2}+1\right) / 2$. Strategies to reduce this figure are discussed below.

The off-resonance behavior of the DPPA is rich and depends on the dimensionless quantities $\kappa_{i} / \Gamma_{m, j}, \mathcal{C}_{1}, \mathcal{C}_{2}$. We plot forward gain, reverse gain, added noise, and the noise spectrum at cavity 1 as functions of frequency at cooperativities $\mathcal{C}_{1}=\{1,3,10,30\}, \mathcal{C}_{2}=\mathcal{C}_{1}-$ $0.1 \sqrt{\mathcal{C}_{1}}$ in Figs. 2(c)-2(f). $\mathcal{C}_{2}$ is chosen such that when increasing $\mathcal{C}_{1}$ both gain and bandwidth are enhanced.

For $\Gamma_{m, j}=\Gamma_{m}, \kappa_{i}=\kappa$, and $\kappa / \Gamma_{m} \gg\left\{1, \mathcal{C}_{1}, \mathcal{C}_{2}\right\}$, gain bandwidth $\Gamma=4 \sqrt{\mathcal{C}_{1} \mathcal{C}_{2}} \Gamma_{m}$ [cf. Fig. 2(c)] [39]. As the gain gets large and $\mathcal{C}_{1}, \mathcal{C}_{2}$ dominate all other dimensionless parameters, the bandwidth approaches $\Gamma=\kappa\left(\mathcal{C}_{1}-\mathcal{C}_{2}\right) / \mathcal{C}_{1}$, leading to the gain-bandwidth product limit $P \equiv \Gamma \sqrt{\mathcal{G}} \rightarrow 2 \kappa$, independent of $\kappa / \Gamma_{m}$. Close to resonance, the reverse-scattering amplitude $S_{a_{2} \rightarrow a_{1}}(\omega) \approx-i \omega \sqrt{\mathcal{G}} / \Gamma_{m}$ [39], such that product of isolation bandwidth and gain is $\Gamma_{m}$. Since gain bandwidth is larger than the isolation 


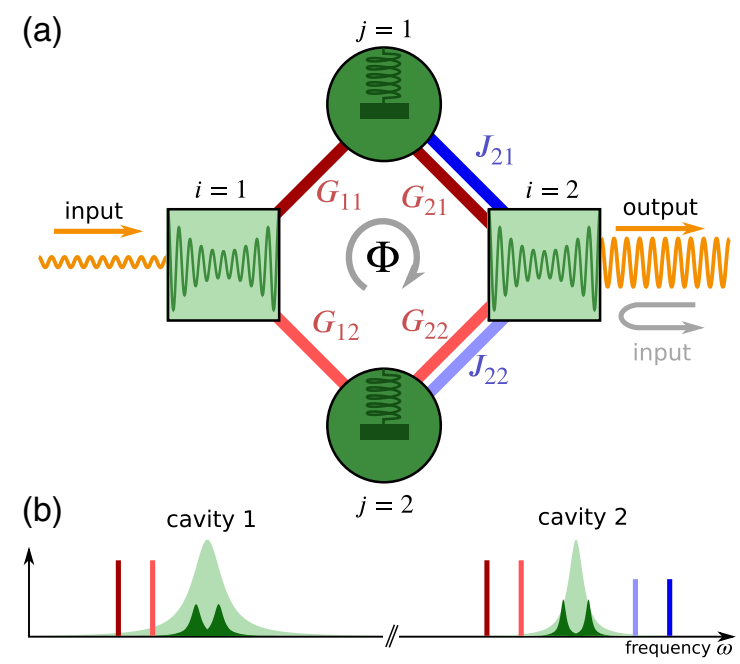

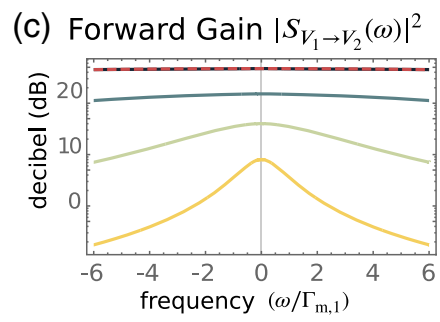

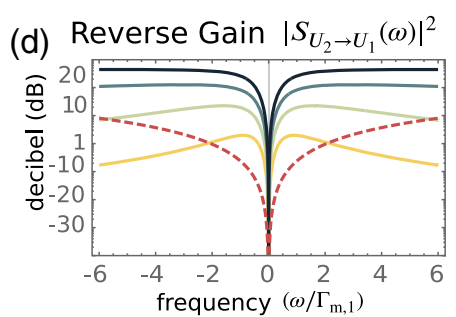

(e)

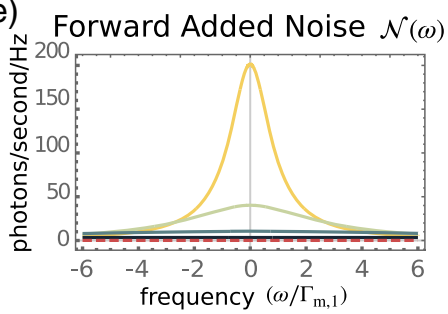

(f)

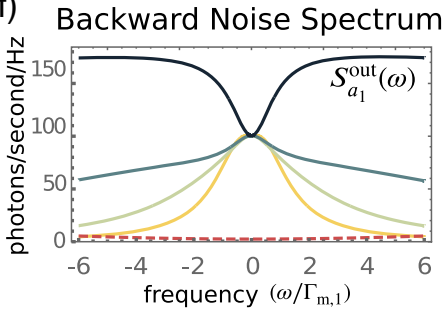

FIG. 3. The directional phase-sensitive amplifier. (a) Model: Single red hopping interactions are impedance matched, double red-blue provide the phase-sensitive amplification. (b) Pump setup: cavity 1 is pumped on the red sidebands of the mechanical resonators, whereas cavity 2 has pumps on the red and blue sidebands. (c)-(f) Plots of forward gain, reverse gain [cf. Eq. (7)], added noise [Eq. (9)], and the output noise fluctuation spectrum of cavity 1 , all as functions of frequency in units of $\Gamma_{m, 1}$, for cooperativities $\mathcal{C}_{1}=\{1,3,10,30\}, \mathcal{C}_{2}=\mathcal{C}_{1}^{2}$ (yellow to black, or light to dark). The parameters are the same as in Fig. 2. Depending on parameters, external sideband cooling with an auxiliary mode can achieve $n_{m, j} \approx 0$, without negatively affecting amplification properties, as described below. The red (dashed) curve in each plot illustrates this case, with $\mathcal{C}_{1}=30$ and effective parameters $n_{\text {eff, } i}=$ $n_{m, i}\left(\Gamma_{m, i} / \Gamma_{\mathrm{eff}, i}\right), \Gamma_{\mathrm{eff}, i}=50 \Gamma_{m, i}$.

bandwidth, there is large reverse gain off resonance [cf. Fig. 2(d)], and noise from cavity 2 dominates the noise spectral density at cavity 1 [Fig. 2(f)]. With increasing effective mechanical linewidth $\Gamma_{m}$ (e.g. through additional sideband cooling), the isolation bandwidth grows, suppressing reverse gain off resonance (cf. red dashed curve in Fig. 2 and Ref. [18]). In the SM we calculate how off-resonant terms renormalize the parameters of the DPPA [39].

Directional phase-sensitive amplifier (DPSA).-We now turn to an implementation of a DPSA, which necessitates six tones. Essentially, we replace the amplifier interaction in the DPPA by a phase-sensitive quantum nondemolition (QND) interaction that couples one quadrature of cavity 2 to only one quadrature of the mechanical resonator $[45,46]$, choosing

$$
\mathbf{G}=\frac{1}{2}\left(\begin{array}{cc}
e^{i \Phi / 2} \sqrt{\mathcal{C}_{1} \Gamma_{m, 1} \kappa_{1}} & e^{-i \Phi / 2} \sqrt{\mathcal{C}_{1} \Gamma_{m, 2} \kappa_{1}} \\
\sqrt{\mathcal{C}_{2} \Gamma_{m, 1} \kappa_{2}} & \sqrt{\mathcal{C}_{2} \Gamma_{m, 2} \kappa_{2}}
\end{array}\right),
$$

and the same $\mathbf{J}$ as for the DPPA [Eq. (5)], illustrated in Figs. 3(a) and 3(b). Since the QND interaction requires $\left|G_{i 2}\right|=\left|J_{i 2}\right|$, and we require symmetric amplifier arms, two cooperativities suffice to characterize the six tones. In the DPSA, we need to ensure the transmitted mechanical quadratures agree, $\quad \mu=\arg \left[G_{11} \chi_{m, 1}(0) G_{21} / J_{21}\right]=$ $\arg \left[ \pm G_{12} \chi_{m, 2}(0) G_{22} / J_{22}\right]$, and that information from both emerges in the same cavity quadrature, $\nu=$ $\arg \left(G_{21} J_{21}\right)=\arg \left( \pm G_{22} J_{22}\right) . \quad \mu$ and $\nu$ determine the quadratures involved in amplification. The two remaining phases are an arbitrary mechanical phase and the plaquette phase.

While there is no parametric instability of the kind that limits backaction-evading measurements [47,48], we show in the SM [39] using a Floquet technique [49,50] that counterrotating terms induce an instability threshold for finite sideband parameter (similar to Ref. [51]), and the RWA is only valid for sideband parameters that are bigger than the cooperativities. This is not out of reach [52], but needs to be taken into account in experimental design.

The isolation, detuning, and impedance-matching conditions coincide with those of the DPPA, and we obtain another central result, the scattering matrix (on resonance)

$$
\begin{aligned}
\left(\begin{array}{l}
U_{1, \text { out }} \\
V_{1, \text { out }} \\
U_{2, \text { out }} \\
V_{2, \text { out }}
\end{array}\right)= & \left(\begin{array}{cccc}
0 & 0 & 0 & 0 \\
0 & 0 & 0 & 0 \\
0 & 0 & -1 & 0 \\
0 & \sqrt{\mathcal{G}} & 0 & -1
\end{array}\right)\left(\begin{array}{l}
U_{1, \text { in }} \\
V_{1, \text { in }} \\
U_{2, \text { in }} \\
V_{2, \text { in }}
\end{array}\right) \\
& +\frac{1}{\sqrt{2}}\left(\begin{array}{cccc}
1 & 0 & -1 & 0 \\
0 & 1 & 0 & -1 \\
0 & 0 & 0 & 0 \\
\sqrt{2 \mathcal{F}} & 0 & \sqrt{2 \mathcal{F}} & 0
\end{array}\right)\left(\begin{array}{c}
X_{1, \text { in }} \\
P_{1, \text { in }} \\
X_{2, \text { in }} \\
P_{2, \text { in }}
\end{array}\right),
\end{aligned}
$$

where we defined noise scattering intensity $\mathcal{F} \equiv 4 \mathcal{C}_{2} / \mathcal{C}_{1}^{2}$, gain 


$$
\mathcal{G}=\frac{8 \mathcal{C}_{2}\left(2 \mathcal{C}_{1}-1\right)}{\mathcal{C}_{1}^{2}}
$$

mechanical $X_{i}=\left(b_{i}+b_{i}^{\dagger}\right) / \sqrt{2}, P_{i}=i\left(b_{i}^{\dagger}-b_{i}\right) / \sqrt{2}$, and optical quadratures $U_{i}=\left(a_{i}+a_{i}^{\dagger}\right) / \sqrt{2}, V_{i}=i\left(a_{i}^{\dagger}-a_{i}\right) / \sqrt{2}$.

The amplifier is phase sensitive and directional, as only the phase quadrature of the second cavity, $V_{2}$, inherits the amplified signal from the phase quadrature of the first cavity, $V_{1}$. We calculate the noise added to the signal as before,

$\mathcal{N}_{\text {DPSA }}=\frac{n_{m, 1}+n_{m, 2}+1}{2\left(2 \mathcal{C}_{1}-1\right)}+\frac{\mathcal{C}_{1}}{8 \mathcal{C}_{2}} \frac{\mathcal{C}_{1}}{2 \mathcal{C}_{1}-1}\left(n_{c, 2}+\frac{1}{2}\right)$.

The crucial difference to DPPA is that the noise stemming from reflection of fluctuations at cavity 2 can also be suppressed, such that in the limit $\mathcal{C}_{2} \gg \mathcal{C}_{1} \gg 1$ added noise vanishes.

We again plot forward gain, reverse gain, added noise, and spectral noise density at cavity 1 in Figs. 3(c)-3(f) at cooperativities $\mathcal{C}_{1}=\{1,3,10,30\}, \mathcal{C}_{2}=\mathcal{C}_{1}^{2}$. Increasing $\mathcal{C}_{1}$ enhances bandwidth and gain [cf. Fig. 3(c)] and suppresses mechanical noise [cf. Fig. 3(e)]. Close to resonance, the reverse scattering behaves like in the DPPA $S_{a_{2} \rightarrow a_{1}}(\omega) \approx$ $-i \omega \sqrt{\mathcal{G}} / \Gamma_{m}$ [39], and the same conclusions apply [cf. Figs. 3(d) and 3(f)]. The gain-isolation-bandwidth product is $\Gamma_{m}$. Forward and reverse gain are proportional to $\sqrt{\mathcal{C}_{2}}$, implying an unlimited gain-bandwidth product [39]. For equivalent mechanical resonators $\Gamma_{m, 1}=\Gamma_{m, 2}=$ $\Gamma_{m}$ and in the limit $\kappa / \Gamma_{m} \gg\left\{1, \mathcal{C}_{1}\right\}$, the amplitude gain bandwidth of the DPSA is well approximated by $\Gamma_{\text {gain }}=2 \mathcal{C}_{1} \Gamma_{m}$

Backward-propagating noise and sideband cooling.The noise emitted in the reverse direction is of central importance for directional amplifiers. For both DPPA and DPSA, the output noise spectral density of cavity 1 on resonance is $S_{1, \mathrm{DPSA}}^{\text {out }}(0)=S_{1, \mathrm{DPPA}}^{\text {out }}(0)=\left(n_{m, 1}+n_{m, 2}+1\right) / 2$. Because of impedance matching and directionality, fluctuations incident on the cavities do not appear in $a_{1 \text {,out }}$ [cf. Eqs. (7) and (11)]. The commutation relations of $a_{1, \text { out }}$ then imply $\sum_{j=1}^{2}\left[\left|S_{b_{i} \rightarrow a_{1}}(0)\right|^{2}-\left|S_{b_{i}^{\dagger} \rightarrow a_{1}}(0)\right|^{2}\right]=1$; i.e., mechanical fluctuations have to appear in the output instead. The lowest possible value for $S_{1 \text {,out }}$ is $1 / 2$, attainable for zero thermal noise quanta in the mechanical resonators.

However, even in state-of-the-art dilution refrigerators, the required temperatures are out of reach. One way to mitigate backward noise emission is to add another microwave mode to the setup that can replace the fluctuations in the output of cavity 1 , essentially realizing a circulator. Without modifying the theory above, one can either increase the mechanical resonator frequencies, which is mainly a technological challenge, or one could resort to external sideband cooling with an auxiliary mode. The latter can achieve $n_{m} \rightarrow 0$ [53-55], and has the added benefit of enhancing mechanical linewidths [cf. red (dashed) curve in Figs. 2 and 3 and SM [39] ]. While this could be done with an additional cavity mode for each resonator, implementing a circuit with four cavity modes coupled to two mechanical resonators is a formidable technical challenge. A problem arises when cooling with only one additional mode, since it can lead to a coupling of the mechanical resonators via the extra cooling mode; this thereby changes the topology of the system, thus spoiling directionality. This can be mitigated by detuning each pump by several mechanical linewidths [39], making cooling with only one additional mode feasible.

Conclusion.-We have presented quantum-limited, nonreciprocal amplifiers using an optomechanical plaquette comprising two cavities and intermediate mechanical resonators $[25,26]$. Such devices carry great promise, since they can be integrated into superconducting circuits and amplify near or at the quantum limit, while protecting the signal source.

We are grateful to John Teufel and, in particular, Anja Metelmann for insightful discussions and helpful comments. D. M. acknowledges support by the UK Engineering and Physical Sciences Research Council (EPSRC) under Grant No. EP/M506485/1. This work was supported by the SNF, the NCCR Quantum Science and Technology (QSIT), and the European Union's Horizon 2020 research and innovation programme under Grant No. 732894 (FET Proactive HOT). T. J. K. acknowledges financial support from an ERC Advanced Grant (QuREM). A. N. received support from a University Research Fellowship from the Royal Society and acknowledges support from the Winton Programme for the Physics of Sustainability.

[1] K. Gallo, G. Assanto, K. R. Parameswaran, and M. M. Fejer, Appl. Phys. Lett. 79, 314 (2001).

[2] J. Koch, A. A. Houck, K. L. Hur, and S. M. Girvin, Phys. Rev. A 82, 043811 (2010).

[3] K. Fang, Z. Yu, and S. Fan, Nat. Photonics 6, 782 (2012).

[4] D. L. Sounas, C. Caloz, and A. Alù, Nat. Commun. 4, 2407 (2013).

[5] A. Kamal, J. Clarke, and M. H. Devoret, Nat. Phys. 7, 311 (2011).

[6] M. Hafezi and P. Rabl, Opt. Express 20, 7672 (2012).

[7] C. G. Poulton, R. Pant, A. Byrnes, S. Fan, M. J. Steel, and B. J. Eggleton, Opt. Express 20, 21235 (2012).

[8] S. Longhi, Opt. Lett. 38, 3570 (2013).

[9] K. Fang, Z. Yu, and S. Fan, Phys. Rev. B 87, 060301 (2013).

[10] D.-W. Wang, H.-T. Zhou, M.-J. Guo, J.-X. Zhang, J. Evers, and S.-Y. Zhu, Phys. Rev. Lett. 110, 093901 (2013). 
[11] B. Abdo, K. Sliwa, S. Shankar, M. Hatridge, L. Frunzio, R. Schoelkopf, and M. Devoret, Phys. Rev. Lett. 112, 167701 (2014).

[12] A. V. Poshakinskiy and A. N. Poddubny, Phys. Rev. Lett. 118, 156801 (2017).

[13] K. M. Sliwa, M. Hatridge, A. Narla, S. Shankar, L. Frunzio, R. J. Schoelkopf, and M. H. Devoret, Phys. Rev. X 5, 041020 (2015).

[14] F. Lecocq, L. Ranzani, G. A. Peterson, K. Cicak, R. W. Simmonds, J. D. Teufel, and J. Aumentado, Phys. Rev. Applied 7, 024028 (2017).

[15] B. Abdo, K. Sliwa, L. Frunzio, and M. Devoret, Phys. Rev. X 3, 031001 (2013).

[16] L. Ranzani and J. Aumentado, New J. Phys. 16, 103027 (2014).

[17] L. Ranzani and J. Aumentado, New J. Phys. 17, 023024 (2015).

[18] A. Metelmann and A. A. Clerk, Phys. Rev. X 5, 021025 (2015).

[19] K. Fang, J. Luo, A. Metelmann, M. H. Matheny, F. Marquardt, A. A. Clerk, and O. Painter, Nat. Phys. 13, 465 (2017).

[20] A. Kamal and A. Metelmann, Phys. Rev. Applied 7, 034031 (2017).

[21] A. Metelmann and A. A. Clerk, Phys. Rev. A 95, 013837 (2017).

[22] Z. Shen, Y.-L. Zhang, Y. Chen, C.-L. Zou, Y.-F. Xiao, X.-B. Zou, F.-W. Sun, G.-C. Guo, and C.-H. Dong, Nat. Photonics 10, 657 (2016).

[23] F. Ruesink, M.-A. Miri, A. Alù, and E. Verhagen, Nat. Commun. 7, 13662 (2016).

[24] X.-W. Xu, Y. Li, A.-X. Chen, and Y.-x. Liu, Phys. Rev. A 93, 023827 (2016).

[25] N. R. Bernier, L. D. Tóth, A. Koottandavida, M. A. Ioannou, D. Malz, A. Nunnenkamp, A. K. Feofanov, and T. J. Kippenberg, Nat. Commun. 8, 604 (2017).

[26] G. A. Peterson, F. Lecocq, K. Cicak, R. W. Simmonds, J. Aumentado, and J. D. Teufel, Phys. Rev. X 7, 031001 (2017).

[27] L. Tian and Z. Li, Phys. Rev. A 96, 013808 (2017).

[28] F. Massel, T. T. Heikkilä, J.-M. Pirkkalainen, S. U. Cho, H. Saloniemi, P. J. Hakonen, and M. A. Sillanpää, Nature (London) 480, 351 (2011).

[29] A. Metelmann and A. A. Clerk, Phys. Rev. Lett. 112, 133904 (2014).

[30] A. Nunnenkamp, V. Sudhir, A. K. Feofanov, A. Roulet, and T. J. Kippenberg, Phys. Rev. Lett. 113, 023604 (2014).

[31] L. D. Tóth, N. R. Bernier, A. Nunnenkamp, A. K. Feofanov, and T. J. Kippenberg, Nat. Phys. 13, 787 (2017).

[32] C. F. Ockeloen-Korppi, E. Damskägg, J.-M. Pirkkalainen, T. T. Heikkilä, F. Massel, and M. A. Sillanpää, Phys. Rev. X 6, 041024 (2016).

[33] J. Suh, A. J. Weinstein, C. U. Lei, E. E. Wollman, S. K. Steinke, P. Meystre, A. A. Clerk, and K. C. Schwab, Science 344, 1262 (2014).

[34] J.-M. Pirkkalainen, E. Damskägg, M. Brandt, F. Massel, and M. A. Sillanpää, Phys. Rev. Lett. 115, 243601 (2015).
[35] E. E. Wollman, C. U. Lei, A. J. Weinstein, J. Suh, A. Kronwald, F. Marquardt, A. A. Clerk, and K. C. Schwab, Science 349, 952 (2015).

[36] C. U. Lei, A. J. Weinstein, J. Suh, E. E. Wollman, A. Kronwald, F. Marquardt, A. A. Clerk, and K. C. Schwab, Phys. Rev. Lett. 117, 100801 (2016).

[37] A. A. Clerk, M. H. Devoret, S. M. Girvin, F. Marquardt, and R. J. Schoelkopf, Rev. Mod. Phys. 82, 1155 (2010).

[38] M. Aspelmeyer, T. J. Kippenberg, and F. Marquardt, Rev. Mod. Phys. 86, 1391 (2014).

[39] See Supplemental Material at http://link.aps.org/ supplemental/10.1103/PhysRevLett.120.023601 for detailed calculations concerning the derivation of the Hamiltonian, the parameter choices, the DPPA with only blue tones, bandwidths, the effect of off-resonant and counterrotating terms, and the relation to other nonreciprocity schemes.

[40] C. W. Gardiner and M. J. Collett, Phys. Rev. A 31, 3761 (1985).

[41] C. Gardiner and P. Zoller, Quantum Noise: A Handbook of Markovian and Non-Markovian Quantum Stochastic Methods with Applications to Quantum Optics, Springer Series in Synergetics (Springer, New York, 2004).

[42] This condition causes the moduli of the transmission amplitudes via resonator 1 and 2 to coincide, $\left|G_{11} J_{21} \chi_{m, 1}(0)\right|=\left|G_{12} J_{22} \chi_{m, 2}(0)\right|$, needed for complete destructive interference.

[43] A. Metelmann (private communication). Note that choosing $G_{21}=J_{22}=0, G_{11}=J_{12}, \delta=0$, yields reciprocal amplifier with unlimited gain-bandwidth product [29].

[44] C. M. Caves, Phys. Rev. D 26, 1817 (1982).

[45] V. B. Braginsky, Y. I. Vorontsov, and K. S. Thorne, Science 209, 547 (1980).

[46] A. A. Clerk, F. Marquardt, and K. Jacobs, New J. Phys. 10, 095010 (2008).

[47] J. B. Hertzberg, T. Rocheleau, T. Ndukum, M. Savva, A. A. Clerk, and K. C. Schwab, Nat. Phys. 6, 213 (2010).

[48] M. J. Woolley and A. A. Clerk, Phys. Rev. A 87, 063846 (2013).

[49] D. Malz and A. Nunnenkamp, Phys. Rev. A 94, 023803 (2016).

[50] D. Malz and A. Nunnenkamp, Phys. Rev. A 94, 053820 (2016).

[51] J. Li, I. M. Haghighi, N. Malossi, S. Zippilli, and D. Vitali, New J. Phys. 17, 103037 (2015).

[52] J. D. Teufel, D. Li, M. S. Allman, K. Cicak, A. J. Sirois, J. D. Whittaker, and R. W. Simmonds, Nature (London) 471, 204 (2011).

[53] J. D. Teufel, T. Donner, D. Li, J. W. Harlow, M. S. Allman, K. Cicak, A. J. Sirois, J. D. Whittaker, K. W. Lehnert, and R. W. Simmonds, Nature (London) 475, 359 (2011).

[54] J. Chan, T. P. M. Alegre, A. H. Safavi-Naeini, J. T. Hill, A. Krause, S. Gröblacher, M. Aspelmeyer, and O. Painter, Nature (London) 478, 89 (2011).

[55] R. Rivière, S. Deléglise, S. Weis, E. Gavartin, O. Arcizet, A. Schliesser, and T. J. Kippenberg, Phys. Rev. A 83, 063835 (2011). 